\title{
Tolerância da placenta à parada circulatória umbilical normotérmica
}

\author{
Renato S. ASSAD *; Fan-Yen LEE **; Joseph SABIK *; Saralyn MACKENZIE **; Frank L. HANLEY **
}

RBCCV 44205-174

ASSAD, R. S.; LEE, F-Y.; SABIK, J.; MACKENZIE, S.; HANLEY, F. L. - Tolerância da placenta à parada circulatória umbilical normotérmica. Rev. Bras. Cir. Cardiovasc., 7(3):157-164, 1992.

RESUMO: O aumento da resistência vascular placentária e distúrbios de trocas gasosas freqũentemente ocorrem após a circulaçāo extracorpórea (CEC) fetal. A utilizaçāo de um circuito convencional de CEC que exclua a placenta da circulação através do clampeamento temporário do cordāo umbilical pode proteger a vasculatura placentária dos estímulos que levam ao aumento da resistência vascular. Para avaliar a tolerância placentária à parada normotérmica do fluxo sangūíneo umbílico-placentário, 9 placentas de ovelhas isoladas in-situ, foram colocadas em CEC através da canulação dos vasos umbilicais, com fluxo médio de artéria umbilical de $214 \mathrm{ml} / \mathrm{min} / \mathrm{kg}$. Após 30 minutos de estabelecimento da CEC, a circulação placentária foi interrompida por 30 minutos, simulando o clapeamento do cordāo umbilical durante a CEC fetal total. Posteriormente, a circulaçāo placentária foi restabelecida aos valores basais. A troca de gases placentária, - fluxo sangüíneo e a resistência vascular materno-placentários foram avaliados antes e depois da parada circulatória. Nāo houve diferença estatísticamente significante entre os gradientes transplacentários de $\mathrm{pO}_{2}$ e $\mathrm{pCO}_{2}$, indicando que a interrupçāo do fluxo sangūíneo placentário por 30 minutos, em condiçōes normotérmicas, não afeta a funçāo placentária. Este modelo sugere um método alternativo para a aplicação clínica da CEC fetal, através da exclusão da circulação umbílico-placentária durante a CEC fetal, eliminandose, assim, os efeitos deletérios da CEC nas trocas gasosas placentárias.

DESCRITORES: circulaçăo extracorpórea fetal; cirurgia fetal; função placentária.

\section{INTRODUÇĀO}

O tratamento cirúrgico intra-uterino de lesōes congênitas, tais como hérnia diafragmática e obstruçōes do trato urinário tem sido aplicado com sucesso no homem ${ }^{12,18}$. A aplicaçāo desta abordagem para determinadas cardiopatias congênitas que se desenvolvem durante a vida fetal, em conseqüência da alteração do padrāo normal de fluxo ou pressāo intracardíacos ${ }^{9,28}$, pode também ser superior ao tratamento cirúrgico neonatal convencional, seja ele paliativo ou definitivo. A circulaçāo extracorpórea (CEC) fetal pode ser necessária para a realização da intervenção intra-uterina. Entretanto, o método ideal de CEC fetal não foi determinado ainda. Estudos da fisiopatologia da CEC fetal demonstram que a disfunçāo placentária constitui o principal fator de morbidade ${ }^{4}$. A CEC fetal convencional, que inclui a perfusão placentária, acarreta um aumento da resistência vascular placentária, a qual determina uma perfusāo placentária inadequada e conseqüentes distúrbios de trocas gasosas, que culminam em óbito fetal 5 .

Com o objetivo de evitar estas alteraçōes placentárias durante a CEC fetal, foi desenvolvida a hipótese de que a exclusão da circulação umbílicoplacentária do circuito da CEC fetal protegeria este leito vascular dos prováveis fatores etiológicos da disfunçāo placentária e, conseqüentemente, da acidose respiratória pós CEC fetal.

Neste estudo, foi utilizada uma preparação de CEC de placentas isoladas, a qual simula a hemodinâmica placentária durante a CEC fetal, para

Trabalho realizado no The Children's Hospital. Departamento de Cirurgia Cardiovascular. Harvard Medical School. Boston, USA.

* Do instituto do Coração do Hospital das Clínicas da Faculdade de Medicina da Universidade de Sáo Paulo. Á época, médico pesquisador no Departamento de Cirurgia Cardiovascular do The Chilcren's Hospital.

** Do The Children's Hospital.

Endereço para separatas: Renato Assad. Av. Dr. Eneas Carvalho de Aguiar,44, Divisão Cirúrgica. 05403, São Paulo, SP, Brasil. 
ASSAD, R. S.; LEE, F-Y.; SABIK, J.; MACKENZIE, S.; HANLEY, F. L. - Tolerância da placenta à parada circulatória umbilical normotérmica. Rev. Bras. Cir. Cardiovasc., 7(3):157-164, 1992.

avaliar a funçăo placentária após 30 minutos de parada circulatória normotérmica do fluxo sangüíneo umbílico-placentário. As trocas gasosas placentárias e o fluxo sangüíneo útero-placentário foram comparados antes e após a parada circulatória.

\section{MATERIAL E MÉTODOS}

\section{1) Preparação do Animal}

Foram estudadas 9 ovelhas prenhas com idade gestacional variando entre 125 a 145 dias. Os animais permaneceram em jejum durante 24 a 48 horas antes do experimento. A anestesia foi induzida com Ketamine I.M. $(20 \mathrm{mg} / \mathrm{kg})$ e mantida com inalaçăo de mistura de $2 \%$ de halotano e $50 \%$ de óxido nitroso. Os vasos femorais foram cateterizados bilateralmente. Um dos cateteres arteriais foi posicionado na aorta torácica e utilizado para injeção de microesferas radioativas. Outros dois cateteres foram colocados em cada artéria femoral e utilizados para monitorizar pressão arterial, hematócrito, gases arteriais, e coletar amostras de referência durante a injeção de microesferas radioativas.
O feto foi exposto através de uma laparotomia mediana infra-umbilical e uma histerotomia de aproximadamente $10 \mathrm{~cm}$ de extensão. Os vasos femorais fetais foram, então, cateterizados, para infusão de drogas e monitorização da pressão arterial fetal. Foi iniciada a infusāo de nitroprussiato de sódio (NTP) através da veia femoral fetal. A dose foi ajustada para reduzir a pressão arterial fetal até $60 \%$ dos valores basais, com o objetivo de suprimir o vasoespasmo associado com a manipulação dos vasos umbilicais.

Uma laparotomia paraumbilical fetal foi realizada para se expor e isolar os vasos umbilicais dentro da cavidade abdominal. Após a dissecção, os vasos umbilicais foram canulados em direção à placenta. Este procedimento ocasionou o óbito fetal, porém o feto foi mantido no útero.

Todos os animais receberam cuidados de acordo com o "Guia de Cuidados e Uso de Animais de Laboratório", preparado pela Academia Nacional de Ciências dos EUA e publicado pelo Instituto Nacional de Saúde dos EUA

O protocolo experimental foi revisado e aprovado pela comissāo de cuidados e uso de animais de laboratório do Children's Hospital, de Boston.

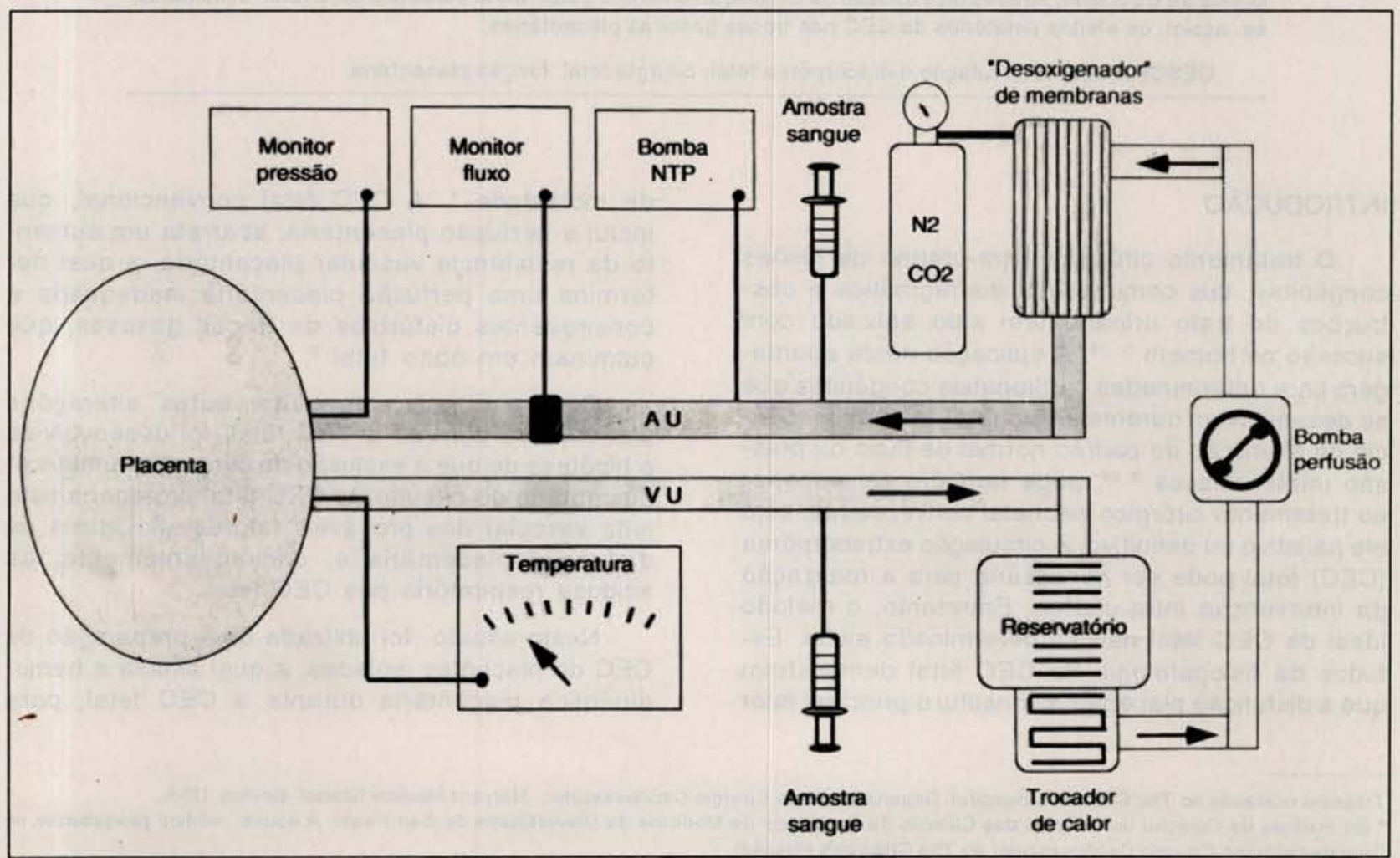

Fig. 1 - Representaçào esquemática do circuito da circulaçào extracorpórea do isolamento placentário NTP: Nitroprussiato de sódio; AU: artéria umbilical; VU: veia umbilical. 
ASSAD, R. S.; LEE, F-Y.; SABIK, J.; MACKENZIE, S.; HANLEY, F. L. - Tolerância da placenta à parada circulatória umbilical normotérmica. Rev. Bras. Cir. Cardiovasc., 7(3):157-164, 1992.

\section{2) Circuito Extracorpóreo}

Após a canulação dos vasos umbilicais, a circulação placentária foi imediatamente restabelecida através de um circuito de CEC (Figura 1) com um fluxo médio de $214 \mathrm{ml} / \mathrm{min} / \mathrm{kg}$ de peso fetal, similar ao fluxo fisiológico da artéria umbilical. $O$ nitroprussiato de sódio foi utilizado novamente na linha arterial do circuito da CEC, com uma dose ajustada para se obter pressão normal de artéria umbilical. Quando a dose ideal foi atingida, a velocidade de infusāo foi mantida constante.

Após a heparinizaçāo dá ovelha $(2 \mathrm{mg} / \mathrm{kg})$, foram utilizados $700 \mathrm{ml}$ de sangue venoso materno como solução de priming. Como o sangue materno apresenta o hematócrito em torno de $25 \%$, sua utilização proporcionou uma hemodiluiçāo moderada satisfatória para a CEC. Foi utilizado oxigenador de membranas infantil (Cobe Laboratories, Inc., Lakewood, EUA) no circuito para ajuste da temperatura, $\mathrm{pO}_{2}, \mathrm{pCO}_{2}$. Um tanque composto de $5 \%$ de $\mathrm{CO}_{2}$ e $95 \%$ de $\mathrm{N}_{2}$ foi utilizado para manter a gasometria arterial dentro dos limites fisiológicos do feto. O fluxo arterial da CEC foi medido com um transdutor eletromagnético (MVF-3100, Nihon Kohden, Tóquio, Japão) e a pressão de perfusão placentária foi medida através de um cateter (22 G) inserido no lume de uma das cânulas arteriais e conectado a um transdutor pré-calibrado (Stathan P23ID, Gould, EUA). O retorno venoso das veias umbilicais era obtido através de simples sifonagem para um reservatório. A CEC foi realizada em normotermia, com fluxo não pulsátil.

\section{3) Injeção de Microesferas}

Microesferas radioativas com atividade específica de $10 \mathrm{mCi} / \mathrm{g}$ foram injetadas no cateter da aorta torácica de 6 ovelhas. As microesferas apresentavam um diâmetro de $14 \pm 1$ microns e eram marcadas com um dos seguintes radioisótopos: ${ }^{46} \mathrm{Sc}$, ${ }^{85} \mathrm{Sr},{ }^{141} \mathrm{Ce},{ }^{125} \mathrm{I}$, or ${ }^{113} \mathrm{Sn}$ (3-M Company e New England Nuclear, EUA). Cada injeção de microesferas consistia de aproximadamente $2.5 \times 10^{6}$ microesferas, para garantir pelo menos 400 microesferas por grama de tecido placentário ${ }^{15}$. A radioatividade das amostras de referência colhidas simultâneamente das artérias femorais direita e esquerda foi analisada com o objetivo de se avaliar a uniformidade da mistura das microesferas na corrente sangüínea.

A radiação gama da placenta foi registrada através de um contador Compugamma (LKB Wallac 1282, Turku, Finlândia) e um computador "Dell System 200". O fluxo sangüíneo placentário foi calculado conforme previamente descrito ${ }^{14}$. A resistên- cia vascular placentária foi calculada através da razão entre a pressão arterial média materna e o fluxo sangüíneo placentário, de acordo com a lei de Ohm's, modificada para a aplicação de fluxo de líqüidos.

\section{4) Protocolo}

O protocolo experimental foi iniciado 30 minutos após a finalização da preparação cirúrgica, com - fim de garantir a estabilidade da preparaçāo.

As amostras para gasometria foram colhidas simultaneamente da artéria e veia umbilicais logo antes da parada normotérmica da circulação placentária. A circulação extracopórea foi, então, interrompida por $\mathbf{3 0}$ minutos. Após a parada circulatória, a circulação placentária foi restabelecida aos valores basais com um fluxo médio de artéria umbilical de $206 \mathrm{ml} / \mathrm{min} / \mathrm{kg}$.

A função placentária foi novamente avaliada através de análise gasometria dos vasos umbilicais, comparando-se estes resultados aos valores basais.

O fluxo e a resistência vascular útero-placentários foram determinados durante 4 períodos distintos do experimento: condição basal, durante a CEC placentária, 25 minutos após a parada da circulação placentária, e após o restabelecimento da CEC placentária.

Ao final dos procedimentos, a ovelha foi sacrificada e a placenta foi dissecada para análise da radioatividade.

\section{5) Análise Estatística}

Os dados foram avaliados pela análise de variância. A significância estatística foi estabelecida ao nível de $5 \%$. Os valores estão expressos como médias \pm desvio padrăo das médias.

TABELA 1

PRESSĀO ARTERIAL FETAL, HEMATÓCRITO E GASOMETRIA ARTERIAL: CONTROLES

\begin{tabular}{lrl}
\hline Pressāo arterial (mmHg) & Pré NTP: 57.6 & \pm 1.8 \\
& Pós NTP: 36.9 & \pm 1.6 \\
Hematócrito (\%) & 40 & \pm 1.5 \\
$\mathrm{pH}$ & 7.31 & \pm 0.03 \\
$\mathrm{PO}_{2}$ (torr) & $16.3 \pm 1.6$ \\
$\mathrm{PCO}_{2}$ (torr) & $54.0 \pm 4.2$ \\
Saturação de $\mathrm{O}_{2}(\%)$ & $23.0 \pm 4.2$ \\
\hline
\end{tabular}

Valores $=$ média \pm desvio padrão das médias $;$ N $=9 ;$ NTP: Nitroprussiato de sódio 
ASSAD, R. S.; LEE, F-Y.; SABIK, J.; MACKENZIE, S.; HANLEY, F. L. - Tolerância da placenta à parada circulatória umbilical normotérmica. Rev. Bras. Cir. Cardiovasc., 7(3):157-164, 1992.

TABELA 2

DADOS DE GASOMETRIA SANGÜINEA

\begin{tabular}{|c|c|c|c|c|c|c|c|c|c|c|}
\hline & \multicolumn{5}{|c|}{ PRÉ-PARADA } & \multicolumn{5}{|c|}{ PÓS-PARADA } \\
\hline & \multicolumn{2}{|c|}{$A U$} & \multicolumn{2}{|c|}{$V U$} & $V U-A U$ & \multicolumn{2}{|c|}{$A U$} & \multicolumn{2}{|c|}{$V U$} & $V U-A U$ \\
\hline $\mathrm{pH}$ & $7.29 \pm$ & 0.01 & $7.33 \pm$ & 0.01 & & 7.22 & 0.01 & $7.25 \pm$ & 0.01 & \\
\hline $\mathrm{PaO}_{2}$ (torr) & $27.9 \pm$ & 1.9 & $34.9 \pm$ & 2.9 & $+7.0 \pm 1.6$ & 30.2 & 3.3 & $35.5 \pm$ & 3.7 & $+5.3 \pm 1.1$ \\
\hline $\mathrm{PaCO}_{2}$ (torr) & $57.6 \pm$ & 2.6 & $50.7 \pm$ & 2.9 & $-6.9 \pm 1.2$ & $62.9=$ & 2.7 & $55.4 \pm$ & 3.1 & $-7.6 \pm 1.0$ \\
\hline Sat. $\mathrm{O}_{2}(\%)$ & $44.1 \pm$ & 4.5 & $59.9 \pm$ & 5.2 & $+15.8 \pm 3.2$ & 43.7 & 6.0 & $55.4 \pm$ & 5.9 & $+11.7 \pm 2.2$ \\
\hline $\mathrm{H}_{2} \mathrm{CO}_{3}^{2}(\mathrm{mEq} / \mathrm{L})$ & $24 \pm$ & 0.6 & $24.1 \pm$ & 0.8 & & 21.5 & 0.7 & $21.1 \pm$ & 0.7 & \\
\hline$B E$ & $-0.7 \pm$ & 0.8 & $0.0 \pm$ & 1.0 & & -3.9 & 1.1 & $-3.8 \pm$ & 1.0 & \\
\hline
\end{tabular}

$\mathrm{N}=9$; Valores $=$ Média \pm desvio padrâo das médias; $\mathrm{AU}=$ Artéria umbilical; $\mathrm{VU}=\mathrm{Veia}$ umbilical; VU $\cdot \mathrm{AU}=\mathrm{Gradiente}$ transplacentário; Sat. $\mathrm{O}_{2}=$ saturação de oxigênio; $\mathrm{BE}$ = excesso de bases.

\section{RESULTADOS}

A Tabela 1 mostra que os valores de pressão da artéria umbilical, gasometria arterial fetal e hematócrito obtidos antes do isolamento placentário estão dentro dos limites fisiológicos de fetos de ovelhas $^{2,10,16}$. A monitorizaçāo contínua da freqüência cardíaca e pressão arterial materna mostraram que estes parâmetros permaneceram dentro dos limites fisiológicos durante todo o experimento.

\section{1) Gasometria Sangüínea}

Os valores pré-parada de $\mathrm{pH}, \mathrm{PO}_{2} . \mathrm{PCO}_{2}$, saturação de $\mathrm{O}_{2}$ e bicarbonato nas amostras colhidas do circuito da CEC estão nos limites fisiológicos

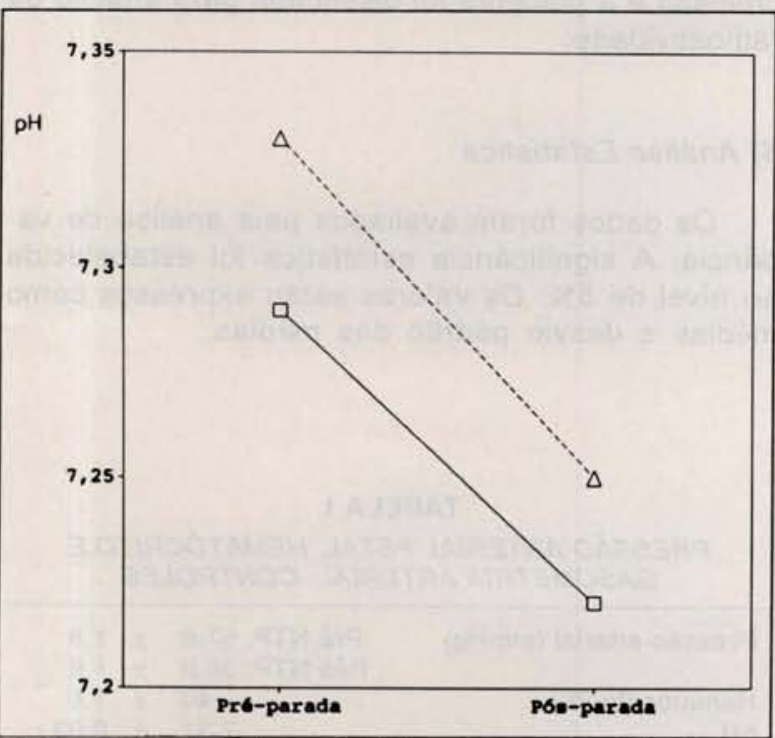

Fig. 2 - Médias dos valores de pH da arteria e veia umbilicais. pre e pós-parada $(n=9)$. O perfusato é significantemente mais acidótico após a parada circulatória normotérmica da placenta $(p<0.01)$

Linha contínua: artéria umbilical; linha pontilhada: veia umbilical. fetais. Após a parada circulatória da placenta, os niveis de gases arteriais permaneceram nos mesmos limites (Tabela 2 e Figuras 2 a 4). Os gradientes transplancentários de $\mathrm{PO}_{2}, \mathrm{PCO}_{2}$ e saturação de $\mathrm{O}_{2}$ permaneceram inalterados após a parada circulatória $(p>0,31)$, com preservação da função placentária. Houve diferença estatísticamente significante dos valores de $\mathrm{pH}$ sangüíneo e bicarbonato entre os períodos de pré e pós-parada circulatória, com certo grau de acidose após a parada $(p<0,01)$.

\section{2) Fluxo Sangüíneo e Resistência Vascular Utero-Placentários}

Os valores basais para estes parâmetros estão

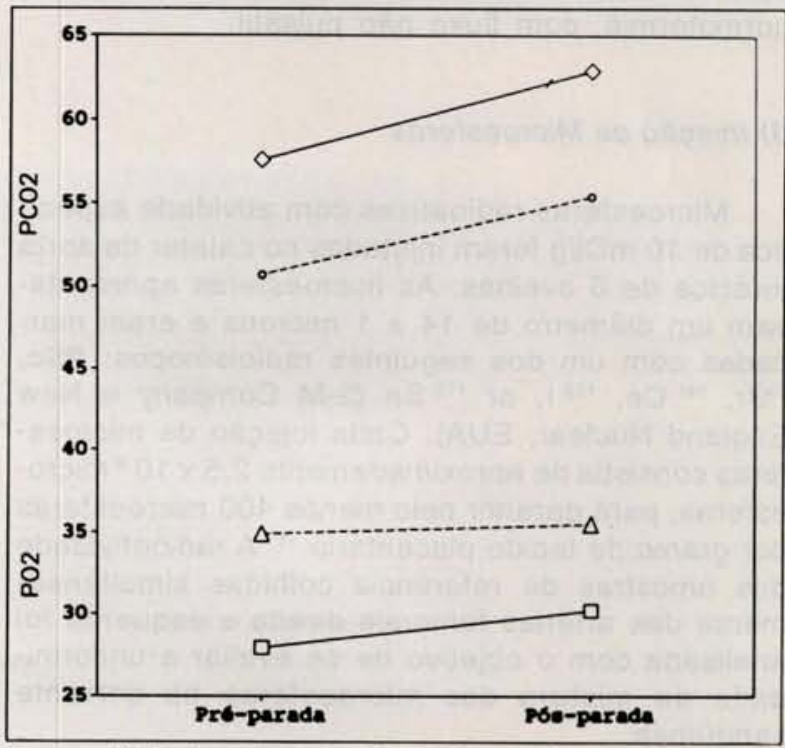

Fig. 3 - Média dos valores de $\mathrm{pO}_{2}$ e $\mathrm{pCO}_{2}$ da artéria e veia umbilicais antes e após a parada circulatória normotérmica da placenta $(n=9)$. Nảo houve diferença estatísticamente significante entre os gradientes transplacentários pré e pós-parada circulatória ( $p>0.39$ ).

Linha contínua: artéria umbilical; linha pontilhada: veia umbilical. 
ASSAD, R. S.; LEE, F-Y.; SABIK, J.; MACKENZIE, S.; HANLEY, F. L. - Tolerância da placenta à parada circulatória umbilical normotérmica. Rev. Bras. Cir. Cardiovasc., 7(3):157-164, 1992.

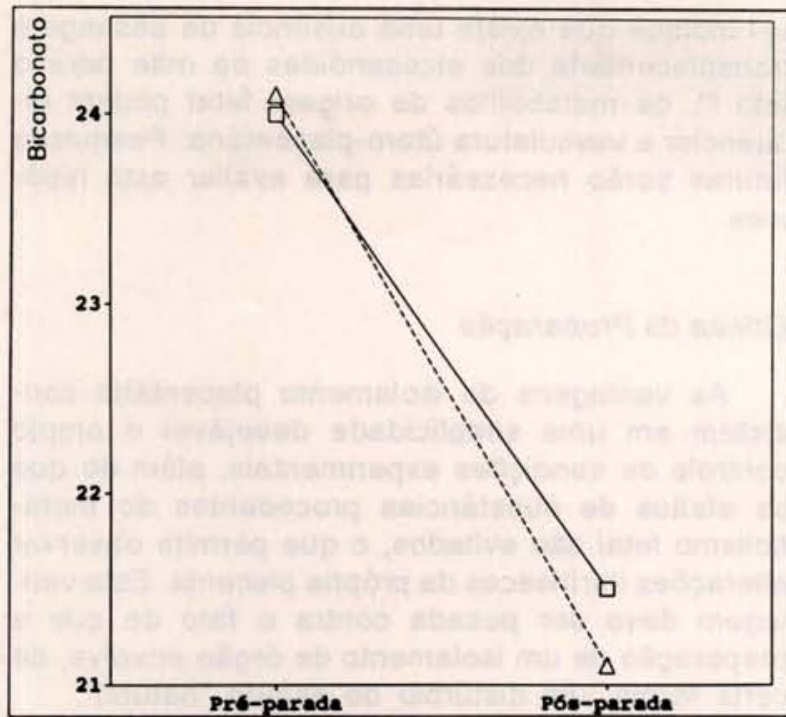

Fig.4. Media dos valores de bicarbonato da arteria e veia umbilicais antes e depois da parada circulatória normotérmica da placenta $(n=9)$. O perfusato apresenta-se significantemente mais acidótico após a parada circulatória $(p<0.02)$.

Linha contínua: artéria umbilical; linha pontilhada: veia umbilical.

nos limites normais das ovelhas prenhas ${ }^{20}$ (Tabela 3 e Figura 5). Durante as condiçōes de CEC placentária, houve significante queda no fluxo sangüíneo útero-placentário $(p=0,03)$, enquanto que o aumento associado da resistência vascular úteroplacentária foi estatísticamente significante apenas após a parada normotérmica da circulaçāo placentária $(p<0,01)$.

Durante a parada normotérmica da circulaçăo, houve certa recuperação destes valores, semelhante aos valores basais $(p=0,21)$, com tendência a valores menores de fluxo sangüíneo e resistência vascular mais elevada.

Os valores de fluxo sangüíneo e resistência vascular útero-placentários após a parada circulatória normotérmica năo săo estatísticamente diferentes dos valores pré-parada $(p>0,41)$, porém com tendência para um menor fluxo placentário após a parada circulatória.

\section{COMENTÁRIOS}

A aplicação clínica da cirurgia cardíaca fetal deve aguardar o desenvolvimento de um circuito de CEC, o qual não induza à disfunção plancetária. Embora o mecanismo desta disfunçāo placentária seja recentemente esclarecido, o estímulo que desencadeia esta resposta indesejável não foi ainda identificado.

A interrupção do fluxo sangüíneo umbílicoplacentário durante a CEC fetal poderia proteger o leito vascular placentário dos efeitos negativos da CEC, evitando-se, assim, a disfunçāo placetária. Isto demandaria o clampeamento dos vasos umbilicais durante a CEC fetal convencional, induzindo

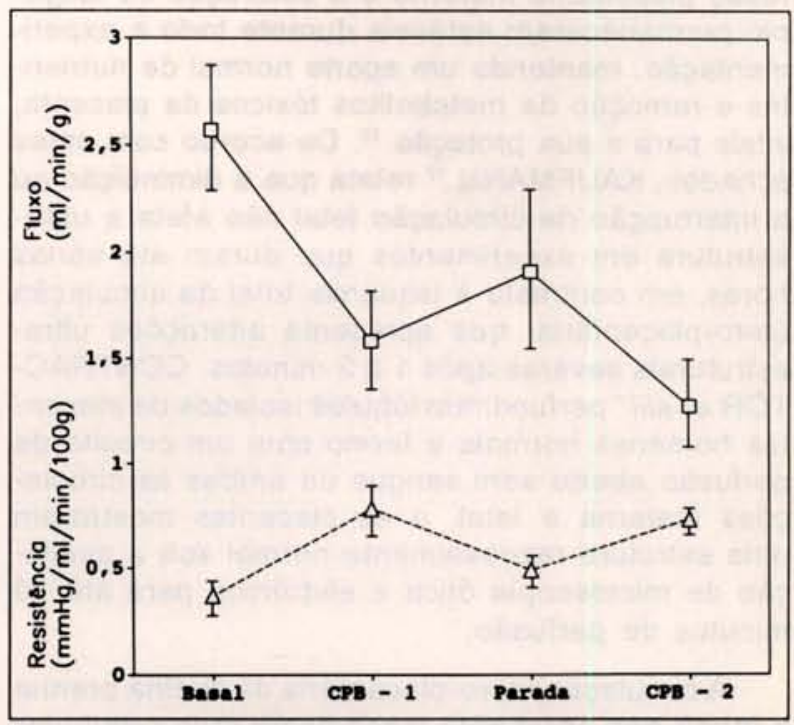

Fig. 5 . Média dos valores \pm desvio padrào das médias do fluxo sangũíneo e resistência vascular útero-placentários durante a condição basal, estabelecimento da CEC placentária, parada normotérmica e após o restabelecimento da perfusão extracorpórea placentária $(n=6)$.

Observe-se uma significante queda do fluxo durante as condiçōes de CEC placentária $(p<0.03)$, enquanto que o aumento associado dos valores de resistência vascular foi estatísticamente significante apenas após a parada circulatória normotérmica ( $p<0.01$ ).

Linha contínua: artéria umbilical; linha pontilhada: veia umbilical.

TABELA 3

DADOS DE FLUXO SANGÜÍNEO E RESISTÊNCIA VASCULAR ÚTERO-PLACENTÁRIOS

\begin{tabular}{lcccc}
\hline & BASAL & CEC - 1 & PARADA & CEC - 2 \\
\hline Fluxo* $^{*}$ & $2.57 \pm 0.27$ & $1.58 \pm 0.29$ & $1.91 \pm 0.41$ & $1.27 \pm 0.21$ \\
Resistência $^{*}$ & $0.37 \pm 0.04$ & $0.78 \pm 0.25$ & $0.49 \pm 0.08$ & $0.73 \pm 0.10$ \\
\hline
\end{tabular}

${ }^{*} \mathrm{ml} / \mathrm{min} / \mathrm{g} ;{ }^{\text {}} \mathrm{mmHg} / \mathrm{ml} / \mathrm{min} / 100 \mathrm{~g} ;$ Valores = Médias \pm desvio padrão das médias; CEC 1 = circulaçăo extracorpórea da placenta pré-parada circulatória; CEC 2 = circulaçảo extracorpórea da placenta pós-parada circulatória. 
ASSAD, R. S.; LEE, F.Y.; SABIK, J.; MACKENZIE, S.; HANLEY, F. L. - Tolerância da placenta à parada circulatória umbilical normotérmica. Rev. Bras. Cir. Cardiovasc., 7(3):157-164, 1992.

à parada circulatória da placenta. A capacidade da placenta em resistir à parada circulatória normotérmica é desconhecida.

Neste estudo, a tolerância da placenta à parada umbílico-placentária foi testada utilizando-se um modelo de isolamento placentário. As trocas gasosas placentárias e o fluxo sangüíneo útero-placentário foram medidos antes e após a parada circulatória umbílico-placentária.

Nossos experimentos demonstram claramente que a função placentária pode ser preservada após 30 minutos de parada normotérmica da circulaçāo umbílico-placentária. As trocas gasosas placentárias nāo evidenciaram prejuízo algum após o período de parada circulatória, um sinal indireto de tecido placentário viável. É importante enfatizar que a perfusāo placentária materna e a saturaçāo de oxigênio permaneceram estáveis durante todo a experimentaçāo, mantendo um aporte normal de nutrientes e remoçāo de metabolitos tóxicos da placenta, vitais para a sua proteção ${ }^{13}$. De acordo com estes achados, KAUFMANN ${ }^{17}$ relata que a diminuiçāo ou a interrupçāo da circulaçăo fetal não afeta a ultraestrutura em experimentos que duram até várias horas, em contraste à isquemia total da circulação útero-placentária, que apresenta alteraçōes ultraestruturais severas após 1 a 2 minutos. CONTRACTOR et alii ${ }^{7}$ perfundiram lóbulos isolados de placentas humanas normais a termo com um circuito de perfusāo aberto sem sangue de ambas as circulaçōes materna e fetal, e as placentas mostraram uma estrutura razoavelmente normal sob a avaliação de microscopia ótica e eletrônica para até 30 minutos de perfusāo.

A circulaçāo útero-placentária da ovelha prenha a termo não apresenta mecanismo auto-regulador 11. A diminuição do fluxo sangüíneo materno-placentário e o aumento na resistência vascular durante as condiçōes de CEC podem estar associados com níveis elevados de substâncias vasoativas, tais como a tromboxane, que podem ser liberadas durante a CEC. Algumas evidências sugerem isto: 1) tem sido demonstrado em publicaçōes anteriores que um aumento importante nos níveis séricos de tromboxane ocorre em pacientes submetidos à cirurgia cardíaca, durante e após a CEC 1, 8, 29;2) a regulação do fluxo sangüíneo em ambos os lados da circulação placentária é altamente dependente do equilíbrio dos metabólitos dos eicosanóides ${ }^{6}$. 19. 24; 3) sabe-se que a tromboxane e a prostaglandina $E_{2}$ causam a vasoconstricçāo placentária $3,19,25,26$.

É possível que um aumento nos níveis de tromboxane durante a CEC fetal possa causar as alteraçōes observadas na circulaçāo útero-placentária. Embora a informação atualmente disponí- vel indique que existe uma ausência de passagem transplacentária dos eicosanóides da mãe para o feto ${ }^{24}$, os metabólitos de origem fetal podem influenciar a vasculatura útero-placentária. Pesquisas futuras serāo necessárias para avaliar esta hipótese.

\section{Crítica da Preparaçāo}

As vantagens do isolamento placentário consistem em uma simplicidade desejável e amplo controle de condiçōes experimentais, além do que os efeitos de substâncias procedentes do metabolismo fetal sảo evitados, o que permite observar alteraçōes intrínsecas da própria placenta. Esta vantagem deve ser pesada contra o fato de que a preparaçāo de um isolamento de órgäo envolve, de certa forma, um distúrbio do estado "natural".

Os dois fatores principais de deterioração da preparaçāo seriam: 1) $\mathrm{O}$ atraso entre a canulaçāo dos vasos umbilicais e o restabelecimento da CEC da perfusăo placentária; 2) os efeitos da circulaçāo extracorpórea em si. No primeiro caso, este fator foi minimizado pela manutençāo deste intervalo entre 3 e 8 minutos. Em relação à $C E C$, foi tentado simular as condiçōes fisiológicas através da perfusão placentária com sangue (materno) e um fluxo normal de artéria umbilical. O trocador de gases no circuito da CEC simulou a respiraçāo dos tecidos, removendo $\mathrm{O}_{2}$ e acrescentando $\mathrm{CO}_{2}$ ao sangue no circuito, a fim de fornecer gases sangüíneos fisiológicos para o leito vascular placentário.

O uso de NTP introduz um elemento não fisiológico neste modelo. Inicialmente, NTP foi administrado para o feto antes da manipulação dos vasos umbilicais para tornar possível a canulação dos mesmos. Posteriormente, o propósito do NTP durante a CEC da placenta foi atingir uma condiçāo inicial tal que a resistência vascular placentária se apresentasse em níveis relativamente normais. Em condiçōes de repouso, a vasculatura placentária apresenta-se próxima da dilataçāo máxima ${ }^{11,21 .}$ Neste estudo, o NTP foi infundido em doses progressivas até que a resistência vascular placentária atingisse níveis normais para um fluxo normal de artéria umbilical, simulando o tonus vascular normal da vasculatura umbílico-placentária em preparaçōes crônicas ${ }^{22,23,27}$. Com uso do NTP, condiçōes basais normais da hemodinâmica placentária foram atingidas.

\section{CONCLUSŌES}

Este trabalho, juntamente com outros estudos 
ASSAD, R. S.; LEE, F-Y.; SABIK, J.; MACKENZIE, S.; HANLEY, F. L. - Tolerância da placenta à parada circulatória umbilical normotérmica. Rev. Bras. Cir. Cardiovasc., 7(3):157-164, 1992.

de alteraçōes estruturais da perfusão de placentas isoladas, indica que uma adequada função placentária pode ser mantida durante a CEC fetal com exclusāo da circulação umbílico-placentária por
30 minutos. Investigaçōes futuras testando esta hipótese com a CEC fetal total serão necessárias para avaliar a aplicação clínica deste método na cirurgia cardíaca fetal.

\section{RBCCV 44205-174}

ASSAD, R. S.; LEE, F. Y.; SABIK, J.; MACKENZIE, S.; HANLEY, F. L. - Tolerance of placenta to normothermic umbilical circulatory arrest. Rev. Bras. Cir. Cardiovasc., 7(3):157-164, 1992.

ABSTRACT: Elevation of placental vascular resistance (PVR) and depression of fetal gas exchange occurs after fetal cardiopulmonary bypass (CPB). Excluding the placenta from the CPB circuit may protect the placental vasculature from the unwanted stimuli which lead to elevated PVR. To evaluate this approach, 9 isolated in-situ sheep placentas were placed on CPB by cannulating the umbilical vessels, with a mean umbilical artery flow of $214 \mathrm{ml} / \mathrm{min} / \mathrm{kg}$. After 30 minutes of stable flow, placental circulation was arrested for 30 minutes, simulating the umbilical vessel clamp time during whole body fetal CPB. Placental circulation was then restored to baseline values. Placental gas exchange and maternal placental blood flow were evaluated before and after arrest. Interruption of blood flow to the placenta for 30 minutes under normothermic conditions does not affect placental function or maternal placental blood flow. It may be possible to exclude the placenta from the circulation during fetal CPB as a mean of eliminating the detrimental effects of CPB on placental gas exchange. This model suggests an alternative way of designing a scheme for clinical fetal CPB which may avoid the negatives effects of CPB on the placenta.

DESCRIPTORS: fetal cardiopulmonary bypass; fetal surgery; placental function.

\section{REFERÊNCIAS BIBLIOGRÁFICAS}

1 ADDONIZIO, V. P.; SMITH, J. B.; STRAUSS, J. F.; COLMAN, R. W.; EDMUNDS, L. H. - Thromboxane synthesis and platelet secretion during cardiopulmonary bypass with bubble oxygenator. $J$. Thorac. Cardiovasc. Surg., 79: 91-96, 1980.

2 ASSALI, N. S.; BRINKMAN, C. R.; NUWAYHID, B. Comparison of maternal and fetal cardiovascular functions in acute and chronic experiments in the sheep. Am. J. Obstet. Gynecol., 120: 411-425, 1974.

BERMAN, W.; GOODLIN, R. C.; HEYMANN, M. A.; RUDOLPH, A. M. - Effects of pharmacologic agents on umbilical blood flow in fetal lambs in utero. Biol. Neonate, 33: 225-235, 1978.

4 BRADLEY, S. M.; HANLEY, F. L.; JENNINGS, R. W.; DUNCAN, B. W.; JESTER, J. A.; VERRIER, E. D. - Regional blood flows during cardiopulmonary bypass in fetal lambs: the effect of nitroprusside. Circulation, 82: III-413, 1990 (Resumo).

BRADLEY, S. M.; VERRIER, E. D.; DUNCAN, B. W.; LONGAKER, M. T.; TURLEY, K.; HARRISON, M. R.; HANLEY, F. L. - Cardiopulmonary bypass in the fetal lamb: effect of sodium nitroprusside. Cir-

culation, 80: II-220, 1989 (Resumo).

6

7 CONTRACTOR, S. F.; EATON, B. M.; FIRTH, J. A.; BAUMAN, K. F. - A comparison of the effects of different perfusion regimes on the structure of the isolated human placental lobule. Cell. Tissue Res., 237: 609-617, 1984.

8 FAYMONVILLE, M. E.; DEBY-DUPONT, G.; LARBUISSON, R.; DEBY, C.; BODSON, L.; LIMET, R.; LAMY, M. - Prostaglandin $E_{2}$, prostacyclin, and thromboxane changes during nonpulsatile cardiopulmonary bypass in humans. J. Thorac. Cardiovasc. Surg., 91: 858-866, 1986.

9 FISHMAN, N. H.; HOF, R. B.; RUDOLPH, A. M.; HEYMANN, M. A. - Models of congenital heart disease in fetal lambs. Circulation, 58:354-364, 1978. - Fetal adaptations to spontaneous hypoxemia and 
ASSAD, R. S.; LEE, F-Y.; SABIK, J.; MACKENZIE, S.; HANLEY, F. L. - Tolerância da placenta à parada circulatória umbilical normotérmica. Rev. Bras. Cir. Cardiovasc., 7(3):157-164, 1992.

responses to maternal oxygen breathing. Biol. Neonate, 46: 276-284, 1984.

11 GREISS, F. C. - Pressure-flow relationships in the gravid uterine vascular bed. Am. J. Obstet. Gynecol., 96: 41-47, 1966.

12 HARRISON, M. R. \& ADZICK, N. S. - The fetus as a patient. Ann. Surg., 213: 279-291, 1991.

13 HEARSE, D. J. - Detection and prevention of tissue injury during hypoxia and ischemia. Contr. Gynecol. Obstet., 13: 48-58, 1985.

14 HEYMANN, M. A.; CREASY, R. K.; RUDOLPH, A. M. - Quantitation of blood flow patterns in the foetal lamb in utero. In: COMLINE, K. S.; CROSS, K. W.; DAWES, G. S.; NATHANIELSZ, P. W. Foetal and neonatal physiology. Cambridge, Cambridge University Press, 1973. p. 129-135.

15 HEYMANN, M. A.; PAYNE, B. D.; HOFFMAN, J. I.; RUDOLPH, A. M. - Blood flow measurements with radionuclide-labeled particles. Prog. Cardiovasc. Dis., 20: 55-79, 1977.

16 IWAMOTO, H. S. \& RUDOLPH, A. M. - Effects of angiotensin II on the blood flow and its distribution in fetal lambs. Circ. Res., 48: 183-189, 1981.

17 KAUFMANN, P. - Influence of ischemia and artificial perfusion on placental ultrastructure and morphometry. Contr. Gynecol. Obstet., 13: 18-26, 1985.

18 LONGAKER, M. T.; COLBUS, M. S.; FILLY, R. A.; ROSEN, M. A.; CHANG, S. W.; HARRISON, M. R. - Maternal outcome after open fetal surgery: a review of the first 17 human cases. JAMA, 265: 737-741, 1991.

19 NOVY, M. J.; PIASECKI, G.; JACKSON, B. T. - Effect of prostaglandins $E_{2}$ and $F_{2}$ on umbilical blood flow and fetal hemodynamics. Prostaglandins, 5: 543555,1974 .

20 PARISI, V. M.; SALINAS, J.; STOCKMAR, E. J. - Placental vascular responses to nicardipine in the hypertensive ewe. Am. J. Obstet. Gynecol., 161: 1039-1043, 1989.
21. PARISI, V. M. \& WALSH, S. W. - Arachidonic acid metabolites and the regulation of placental and other vascular tone during pregnancy. Semin. Perinatol., 10: $288-298,1986$
22
PARISI, V. M. \& WALSH, S. W. - Fetal placental vascular responses to prostacyclin after angiotensin II-induced vasoconstriction. Am. J. Physiol., 257: 102-107, 1989.

PARISI, V. M. \& WALSH, S. W. - Fetal vascular response to prostacyclin. Am. J. Obstet. Gynecol., 160: 871$878,1989$.

PARISI, V. M. \& WALSH, S. W. - Fetoplacental vascular responses to prostacyclin after thromboxane-induced vasoconstriction. Am. J. Obstet. Gynecol., 160: 502-507, 1989.

5 RANKIN, J. H. G. - Role of prostaglandins in the maintenance of the placental circulation. In: COCEANI, F. \& OLLEY, P. M. Advances in proetaglandin and thromboxane research. New York, Raven Press, 1978. p. 221-229.

6 RUDOLPH, A. M. \& HEYMANN, M. A. - Hemodynamic changes induced by blockers of prostaglandin synthesis in the fetal lamb in utero. In: COCEANI, F. \& OLLEY, P. M. Advances in prostaglandin and thromboxane research. New York, Raven Press, 1978. p. 231-237.

7 SCHWARTZ, D. B.; PHERNETTON, T. M.; STOCK, M. K.; RANKIN, J. H. G. - Placental vascular responses to 6-keto-prostaglandin $E_{1}$ in the near-term sheep. Am. J. Obstet. Gynecol., 145: 406-410, 1983.

28 TURLEY, K.; VLAHAKES, G. J.; HARRISON, M. R.; MESSINA, L.; HANLEY, F.; UHLIG, P. N.; EBERT, P. A. - Intrauterine cardiothoracic surgery: the fetal lamb model. Ann. Thorac. Surg., 34: 422-426, 1982.

9 WATKINS, W. D.; PETERSON, M. B.; KONG, D. L.; KONO, K.; BUCKLEY, M. J.; LEVINE, F. H.; PHILBIN, D. M. - Thromboxane and prostacyclin changes during cardiopulmonary bypass with and without pulsatile flow. J. Thorac. Cardiovasc. Surg., 84: 250-256, 1982. 